


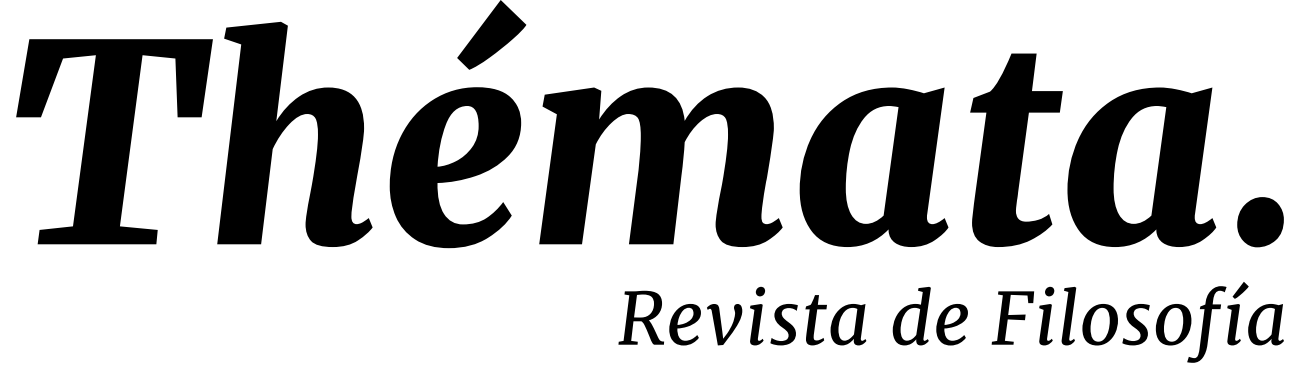

\section{La transmisión filosófica. Pensamiento de Javier Hernández-Pacheco Sanz}

Alejandro Martín Navarro (Coord.)

ESPECIAL THÉMATA

2022

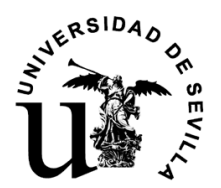

ISSN 0212-8365

e-ISSN 2253-900X

DOI: $10.12795 /$ themata

revistascientificas.us.es/index.php/themata

https://editorial.us.es/es/revistas/themata-revista-de-filosofia 
Thémata. Revista de Filosofía.

Número Extra

Thémata. Revista de Filosofía nace en el año 1983 con la intención de proporcionar a quienes investigan y producen en filosofía un cauce para publicar sus trabajos y fomentar un diálogo abierto sin condicionamientos ideológicos. En sus inicios participaron en el proyecto las Universidades de Murcia, Málaga y Sevilla, pero pronto quedaron como gestores de la revista un grupo de docentes de la Facultad de Filosofía de la Universidad de Sevilla.

Una preocupación constante de sus realizadores ha sido fomentar los planteamientos interdisciplinares. La revista ha estado abierta siempre a colaboradores de todas las latitudes y ha cubierto toda la gama del espectro filosófico, de lo que constituye una buena prueba la extensa nómina de autores que han publicado en sus páginas. En sus páginas pueden encontrarse trabajos de todas las disciplinas filosóficas: Historia de la Filosofía, Metafísica, Gnoseología, Epistemología, Lógica, Ética, Estética, Filosofía Política, Filosofía del Lenguaje, Filosofía de la Mente, Filosofía de la Ciencia, Filosofía de la Historia, Filosofía de la Cultura, etc. También ha querido ser muy flexible a la hora de acoger nuevos proyectos, fomentar discusiones sobre temas controvertidos y abrirse a nuevos valores filosóficos. Por esta razón, los investigadores jóvenes siempre han encontrado bien abiertas las puertas de la revista.

\section{Equipo editorial / Editorial Team Bases de Datos y Repertorios}

Director honorario

Jacinto Choza Armenta

jchoza@us.es

Director

Fernando Infante del Rosal

finfante@us.es

Director Adjunto

José Manuel Sánchez López

themata@us.es

Subdirectores

Jesús Navarro Reyes

jnr@us.es

Inmaculada Murcia Serrano

imurcia@us.es

Jesús de Garay

jgaray@us.es

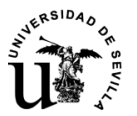

Facultad de Filosofía. Universidad de Sevilla

Departamento de Estética e Historia de la

Filosofía

Departamento de Filosofía y Lógica y Filosofía

de la Ciencia

Departamento de Metafísica y Corrientes

Actuales de la Filosofía, Ética y Filosofía Política

Camilo José Cela s/n, 41018 Sevilla (España)

e-mail: themata@us.es
Bibliográficas internacionales

Emerging Sources Citation Index (Web of

Science Group-Clarivate Analytics)

Dialnet (España)

Francis, Philosophie. INIST-CNRS (France)

Philosopher's Index (Bowling Green, OH, USA)

Directory of Open Access Journals (DOAJ)

Repertoire Bibliographique de Philosophie

(Louvain, Belgique)

Ulrich's Internacional Periodicals Directory

(New York, USA)

DialogJournalNameFinder (Palo Alto, CA, USA)

Periodicals Index Online (Michigan, USA)

Index Copernicus World of Journals

Gale-Cengage Learning-Informe Académico

Academic Journal Database

DULCINEA

Google Scholar

Electra

Bulletin signaletique. Philosophie, CNRS

(France)

Bibliográficas nacionales

ISOC - Filosofía. CINDOC (España)

De evaluación de la calidad de revistas

CARHUS Plus

ERIH PLUS Philosophy (2016)

REDIB

Latindex 2.0

MIAR

CIRC

DICE

Política editorial y directrices para autores/as, al final de la revista.

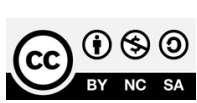


Thémata. Revista de Filosofía.

\section{Consejo Editor / Editorial Board}

\section{ARGENTINA}

Flavia Dezzuto, Universidad Nacional de Córdoba

ALEMANIA

Alberto Ciria, Munich

\section{CANADÁ}

Óscar Moro, University of New Found Land

CHILE

Mariano De la Maza, Universidad Católica de Chile

José Santos Herceg, Universidad de Santiago de Chile

\section{COLOMBIA}

Martha Cecilia Betancur García, Universidad de Caldas

Víctor Hugo Gómez Yepes, Universidad Pontificia Bolivariana

Gustavo Adolfo Muñoz Marín, Universidad Pontificia Bolivariana

\section{ESPAÑA}

Alfonso García Marqués, Universidad de Murcia

Antonio De Diego González, Universidad de Sevilla

Avelina Cecilia Lafuente, Universidad de Sevilla

Carlos Ortiz Landázuri, Universidad de Navarra

Celso Sánchez Capdequí, Universidad Pública de Navarra

Elena Ronzón Fernández, Universidad de Oviedo

Enrique Anrubia, Universidad CEU Cardenal Herrera

Federico Basáñez, Universidad de Sevilla

Fernando Wulff, Universidad de Málaga

Fernando M. Pérez Herranz, Universidad de Alicante

Fernando Pérez-Borbujo, Universitat Pompeu Fabra

Francisco Rodríguez Valls, Universidad de Sevilla

Ildefonso Murillo, Universidad Pontificia de Salamanca

Irene Comins Mingol, Universitat Jaume I Jacinto Rivera de Rosales Chacón, † UNED Joan B. Llinares, Universitat de València Jorge Ayala, Universidad de Zaragoza José Manuel Chillón Lorenzo, Universidad de Valladolid
Juan García González, Universidad de Málaga

Juan José Padial Benticuaga, Universidad de Málaga

Luis Miguel Arroyo Arrayás, Universidad de Huelva

Ma Luz Pintos Peñaranda, Universidad de Santiago de Compostela

Marcelo López Cambronero, Instituto de Filosofía Edith Stein

María del Carmen Paredes, Universidad de Salamanca

Octavi Piulats Riu, Universitat de Barcelona

Óscar Barroso Fernández, Universidad de Granada

Pedro Jesús Teruel, Universitat de València

Ramón Román Alcalá, Universidad de Córdoba

Ricardo Parellada, Universidad Complutense de Madrid

Sonia París Albert, Universitat Jaume I

Tomás Domingo Moratalla, UNED

\section{ESTADOS UNIDOS}

Witold Wolny, University of Virginia)

Thao Theresa Phuong Phan, University of Maryland

\section{REINO UNIDO}

Beatriz Caballero Rodríguez, University of Strathclyde

ITALIA

Luigi Bonanate, Università di Torino

\section{MÉXICO}

Rafael De Gasperín, Instituto Tecnológico de Monterrey

Julio Quesada, Universidad Veracruzana

Adriana Rodríguez Barraza, Universidad Veracruzana

PERÚ

Ananí Gutiérrez Aguilar, Universidad Nacional de San Agustín de Arequipa y Universidad Católica de Santa María

Nicanor Wong Ortiz, Universidad San Ignacio de Loyola

\section{PORTUGAL}

Yolanda Espiña, Universidade Católica Portuguesa

\section{TURQUÍA}

Mehmet Özkan, SETA Foundation for Political, Economic and Social Research 
Thémata. Revista de Filosofía.

\section{Comité Científico Asesor / Advisory Board}

ARGENTINA

Graciela Maturo, Universidad de Buenos Aires - CONICET

Jaime Peire, Universidad Nacional de Tres de Febrero- CONICET

\section{ALEMANIA}

Tomás Gil, Freie Universität Berlin

Fernando Inciarte, $\dagger$ Westfälische WilhelmsUniversität

Otto Saame, † Universität Mainz

\section{BULGARIA}

Lazar Koprinarov, South-West University 'Neofit Rilski'

CHILE

Carla Corduá, Universidad de Chile

Roberto Torreti, Universidad de Chile

COLOMBIA

Carlos Másmela, Universidad de Antioquía Fernando Zalamea, Universidad Nacional de Colombia

\section{ESPAÑA}

Agustín González Gallego, Universitat de Barcelona

Alejandro Llano, Universidad de Navarra Andrés Ortiz-Osés, Universidad de Deusto Ángel D'ors, † Universidad Complutense de Madrid

Antonio Hermosa Andújar, Universidad de Sevilla

Carlos Beorlegui Rodríguez, Universidad de Deusto

Concha Roldán Panadero, Instituto de Filosofía, CCHS-CSIC

Daniel Innerarity Grau, Ikerbasque, Basque Foundation for Science

Francisco Soler, Universidad de Sevilla Ignacio Falgueras, Universidad de Málaga Javier San Martín, UNED

Jesús Arellano Catalán, † Universidad de Sevilla

Joaquín Lomba Fuentes, Universidad de Zaragoza

Jorge Vicente Arregui, † Universidad de Málaga José María Prieto Soler, † Universidad de Sevilla José Rubio, Universidad de Málaga Juan Antonio Estrada Díaz, Universidad de Granada

Juan Arana Cañedo-Argüelles, Universidad de Sevilla
Luis Girón, Universidad Complutense de Madrid

Manuel Fontán Del Junco, Fundación March

Manuel Jiménez Redondo, Universitat de València

Marcelino Rodríguez Donís, Universidad de Sevilla

Miguel García-Baró López, Universidad Pontificia Comillas

Modesto Berciano, Universidad de Oviedo

Pascual Martínez-Freire, Universidad de Málaga

Rafael Alvira, Universidad de Navarra

Teresa Bejarano Fernández, Universidad de Sevilla

Vicente San Félix Vidarte, Universitat de València

\section{ESTADOS UNIDOS}

Lawrence Cahoone, University of Boston

FRANCIA

Nicolás Grimaldi, Université Paris IV-Sorbonne

\section{PARAGUAY}

Mario Ramos Reyes, Universidad Católica de Asunción

\section{REINO UNIDO}

Alexander Broadie, University of Glasgow

ISRAEL

Marcelo Dascal, † Tel Aviv University

ITALIA

Massimo Campanini, Università di Napoli l'Orientale

Maurizio Pagano, Università degli Studi del Piamonte Orientale. Amedeo Avogadro

JAPóN

Juan Masiá, Sophia University, Tokio

\section{MÉXICO}

Jaime Méndez Jiménez, Universidad Veracruzana

Ana Laura Santamaría, Instituto Tecnológico de Monterrey

Héctor Zagal, Universidad Panamericana

VENEZUELA

Seny Hernández Ledezma, Universidad Central de Venezuela

Thémata. Revista de Filosofía agradece la labor realizada desde su inicio por sus anteriores directores: Jacinto Choza, Juan Arana y Jesús de Garay. 


\section{Índice.}

\section{LA TRANSMISIÓN FILOSÓFICA. PENSAMIENTO DE JAVIER HERNÁNDEZ-}

PACHECO SANZ_ESPECIAL THÉMATA

9 El acto filosófico. En recuerdo de Javier Hernández-Pacheco Sanz Fernando Infante del Rosal

14 Presentación

Alejandro Martín Navarro (Coord.)

SEMBLANZAS Y RECUERDOS_JAVIER HERNÁNDEZ-PACHECO SANZ,

TRANSMISIÓN Y ENSEÑANZA DE LA FILOSOFÍA

19 Treinta y siete años con Pacheco. Variaciones sobre el cristianismo Jacinto Choza Armenta

$51 \quad$ Historia de un hombre sabio y bueno Juan Arana Cañedo-Argüelles

$54 \quad$ Una fuerza de la naturaleza José Manuel Sánchez López

58 In Memoriam a Javier Hernández-Pacheco Urbano Ferrer Santos

65 Recordando al profesor Javier Hernández-Pacheco Esperanza María Domínguez Sabido

OBRA Y PENSAMIENTO DE JAVIER HERNÁNDEZ-PACHECO SANZ_ARTÍCULOS

$71 \quad$ Una conversación pendiente Jesús de Garay Suárez-Llanos

87 Un viaje de ida y vuelta: de cómo el refugio se torna apertura. El proyectar(se) como apertura a lo Otro Elisa Fernández Bascones

107 Max Horkheimer y el anhelo de lo completamente Otro. (A propósito de la interpretación de la filosofía de Max Horkheimer de Javier Hernández-Pacheco) José Manuel Panea Márquez 
Thémata. Revista de Filosofía.

124 Javier Hernández-Pacheco: fe y filosofía

Lidia Romero Sánchez

$141 \quad$ La plenitud hacia la que tendemos: historia de una discusión truncada.

Francisco José Soler Gil

161 Tábula gratulatoria.

163 Política editorial.

166 Directrices para autores/as.

ILUSTRACIÓN DE CUBIERTA: Javier Infante 
Thémata. Revista de Filosofía.
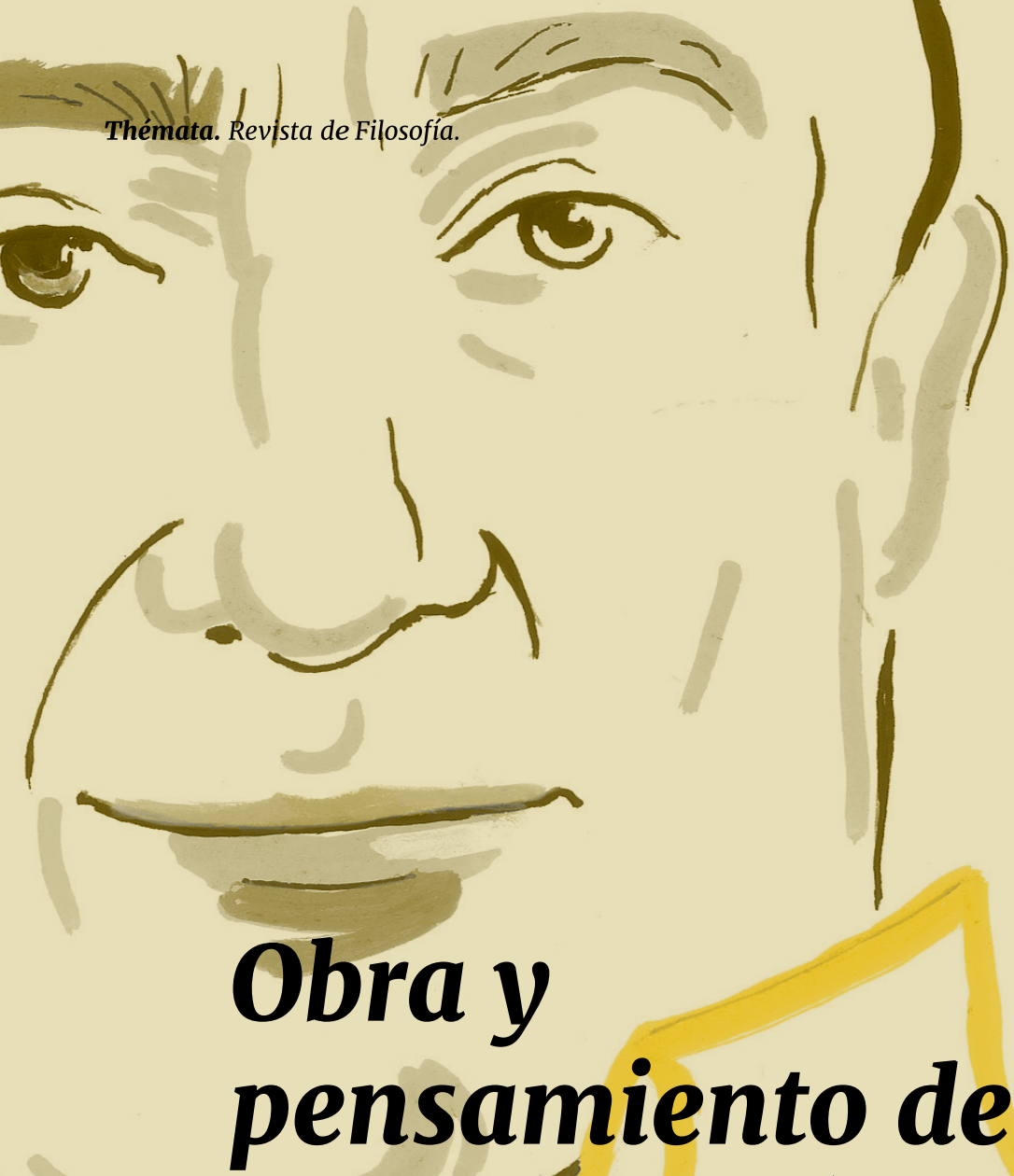

Javier HernándezPacheco Sanz. 


\title{
La plenitud hacia la que tendemos: historia de una discusión truncada.
}

The fullness towards which we tend:

History of a truncated discussion.

\author{
Francisco José Soler Gil' \\ Universidad de Sevilla, España \\ Recibido 1 noviembre 2021 · Aceptado 1 diciembre 2021
}

Resumen

En este artículo se expone en primer lugar una de las tesis centrales del pensamiento de Javier HernándezPacheco: la tesis del imperativo moral que nos impulsa a la transformación del mundo para convertirlo en expresión de la libertad. Y en segundo lugar se resume la discusión que Javier HernándezPacheco y el autor de este artículo sostuvieron acerca de la compatibilidad del fin que se expresa en ese imperativo moral con el marco de la naturaleza expresado por las leyes de la física.

Palabrasclave: Javier Hernández-Pacheco, Fichte, libertad, física, absoluto, filosofía de la naturaleza

\section{Abstract}

This article first exposes one of the central theses of Javier HernándezPacheco's thought: the thesis of the moral imperative that drives us to transform the world to turn it into an expression of freedom. And secondly, the discussion that Javier HernándezPacheco and the author of this article held about the compatibility of the end that is expressed in that moral imperative with the framework of nature expressed by the laws of physics is summarized.

Keywords: Javier Hernández-Pacheco, Fichte, Freedom, Physics, Absolute, Philosophy of Nature 


\section{1・Introducción}

La muerte de Javier Hernández-Pacheco el pasado 17 de noviembre de 2020 ha truncado una década de amistad y de largas, enconadas y gozosas discusiones, que comenzó con mi incorporación a la Facultad de Filosofía de la Universidad de Sevilla, en 2010.

A Javier Hernández-Pacheco lo había conocido ya tres años antes, con ocasión de una visita mía a Sevilla. Pero hasta el comienzo del curso académico en otoño de 2010 no habíamos mantenido más que la relación superficial que suele establecerse entre profesores que contactan con motivo de un congreso, o de un simposio. Nada subrayable.

Lo bueno -ya digo- comenzó en otoño de 2010, cuando me fue asignado justo el despacho contiguo al suyo, y la vecindad se convirtió en ocasión de un intercambio de cortesías convencionales primero, pero pronto también de ideas. De manera que en cuestión más de semanas que de meses ya nos habíamos enzarzado en un debate inagotable, sobre los más diversos asuntos. Una amistad socrática, alimentada por mil discusiones, y que ahora se ha quebrado de forma abrupta y muy amarga.

Quizás podría concretar aún más, y fijar el punto de partida de esa bella relación en torno a un momento muy concreto. Fue el día 22 de noviembre de 2010. Aquel día, a iniciativa de varios compañeros de nuestro departamento, y en especial de Juan Arana y Francisco Rodríguez Valls, pronuncié en el Salón de Grados de la Facultad de Filosofía de Sevilla una "lección inaugural", anunciada bajo el título de "Cosmología: Una disciplina entre la física y la filosofía". Javier Hernández-Pacheco estuvo allí. Y dada nuestra diferente valoración de las posibilidades filosóficas de la física -que yo ya medio conocía (o medio barruntaba) por entonces-, anticipaba de su parte cierta indiferencia, o un posicionamiento más bien crítico... pero ni lo uno ni lo otro: Hernández-Pacheco estuvo allí, y me esperó a la salida del acto. Y aún conservo bien grabada su expresión de alegría al comentarme: "Bien, bien... iMe parece que nos vamos a divertir mucho en este grupo!"

¡Y vaya si nos divertimos! Ahora, cada vez que vuelvo sobre aquel momento, reconozco que fue una profecía que comenzó a verificarse de inmediato. Y la celebro como tal, y como tal me duele, al tener que referirme a su cumplimiento ya cerrado. 
Aún guardo en mi despacho la última botella de whisky (escocés y single malt, por supuesto...), que ya no terminaremos. Y aún guardo una caja de puritos con la que ya no podré tentar su poco firme renuncia al tabaco, con motivo de ciertos problemas de salud que tuvo hace algún tiempo... Pero, sobre todo, guardo memoria de nuestras conversaciones: en su despacho, y en el mío, y de camino hacia Triana. O paseando por el barrio de Santa Cruz, donde tantas veces nos sentábamos a tomar algo en una terracita, al anochecer, cuando volvíamos de la facultad, y lo que iba a ser una cerveza en cinco minutos se convertía en una disputa filosófica gastronómicamente bien pertrechada, que interrumpíamos hacia la media noche, atendiendo a que algo de voz nos quedara para las clases del día siguiente.

$\mathrm{Si}$ a todo esto sumo los miles de emails que cruzamos en un grupo de discusión en el que ambos participamos durante varios años, creo que puedo decir sin exageración que Javier Hernández-Pacheco ha sido para mí no sólo un amigo entrañable, sino la persona que más me ha obligado a pensar y repensar cosas en todo este tiempo. Ha sido un hermano mayor. El hermano que espolea cada día con sus comentarios, que siempre sabe más, y siempre encuentra un enfoque que sorprende, y que muestra caminos abiertos allí donde uno no ve más que contradicciones y complicaciones insalvables.

La sabiduría de Javier Hernández-Pacheco me ha abierto caminos en muchos paisajes que yo tenía por intransitables. Y así, en cuestiones de pensamiento político y económico, por ejemplo, he asumido prácticamente todo lo que él me enseñó. Sin embargo, en asuntos de teología, de ontología y de filosofía de la naturaleza nunca terminamos de ponernos de acuerdo. Y mantuvimos las espadas en alto hasta el final. Por eso, cuando en el fragor de algún debate político, se nos decía que Hernández-Pacheco y yo formábamos una especie de "pinza dialéctica", él a veces respondía sonriendo que nosotros dos estábamos de acuerdo en todo, menos en las cuestiones principales.

Algo de exceso había en esa respuesta - al fin y al cabo, los dos éramos cristianos (... y católicos cristianos, dicho sea de paso)-, pero también había en ella algo de cierto. Y como botón de muestra, me gustaría ofrecerle al lector, en los apartados siguientes, el esbozo de una de esas cuestiones disputadas, que habrá de quedar ya necesariamente en fragmento.

Se trata de un tema que espero que resulte de particular interés para el lector que trata de aproximarse a la obra de Javier Hernández-Pacheco, 
puesto que tiene que ver con una de las claves principales de su pensamiento. Quizás su clave principal: la tarea infinita de transformación del mundo a la que estamos llamados.

Presentaré a grandes rasgos esta idea en el próximo apartado [apartado 2]. A continuación, resumiré la objeción que solía plantearle en nuestras conversaciones al respecto [en el apartado 3]. Esbozaré luego [en el apartado 4] la propuesta de síntesis que yo había ido poco a poco madurando, y que ya no podremos discutir. Y concluiré [en el apartado 5] con unas mínimas reflexiones sobre el carácter fragmentario de esta discusión.

\section{Una clave del pensamiento de Javier Hernández-Pacheco: Nuestra tarea infinita de transformación del mundo}

En consonancia con su desbordante energía, Javier HernándezPacheco fue un autor prolífico. Entre 1983 y 2019 publicó diecisiete libros (lo que proporciona una media de un libro cada dos años, aproximadamente, sostenida a lo largo de un periodo de casi cuatro décadas), más un considerable número de artículos en revistas de filosofía, y otros textos.

$\mathrm{Al}$ acercarnos a esta obra, encontramos de un lado trabajos centrados en el pensamiento de ciertos filósofos (Santo Tomás de Aquino, Hegel, Nietzsche, Heidegger, la Escuela de Frankfurt...), y de otro lado estudios de carácter temático: sobre el romanticismo, la economía, la milicia, etc.

No obstante, la dispersión de un panorama tan amplio queda contrarrestada por ciertos rasgos que dotan a los libros de HernándezPacheco de un carácter propio, reconocible más allá del asunto concreto de cada texto.

Algunos de esos rasgos son estilísticos, y tienen que ver con la eficacia y la agilidad de su escritura, y con su capacidad de integrar de continuo en el discurso giros y expresiones del lenguaje coloquial, que adquieren de repente una puntería y un alcance insospechados. Tales características hacen de las obras de Hernández-Pacheco algo inconfundible. Sus libros hablan con una voz propia, en la que se reconoce al autor a cada momento. Muchos de ellos son incluso libros chispeantes. Y si él recordaba con cierta frecuencia la opinión de Friedrich Schlegel de que la filosofía es Witz, bien puede decirse 
que sus libros tienen justo eso, tan difícil de traducir, y aún más de conseguir: gracia, donaire, ingenio, chispa... Witz.

Pero además del estilo, los libros de Javier Hernández-Pacheco poseen importantes elementos unificadores en lo relativo al contenido. De manera que, pese a la amplitud temática de sus obras, no deja de percibirse en ellas el despliegue y la maduración de unas claves que van siendo utilizadas para el análisis de los distintos temas, y que proporcionan al conjunto un carácter unitario. Más orgánico que sistemático.

Pues bien, una de esas claves orgánicas del pensamiento de Javier Hernández-Pacheco, y sin duda una de las más importantes, es la idea de que el hombre está llamado a una tarea de transformación del mundo: una transformación que lo complete y lo haga habitable, "humano", tanto en el sentido de benigno, como en el de ser obra de nuestra libertad. Una tarea que, de suyo, es infinita, y por tanto incompletable desde nuestra finitud. Pese a lo cual impera en nuestra conciencia el mandato de esforzarnos en su ejecución, con la esperanza de que el infinito faltante sea recibido como regalo, en el horizonte escatológico de esa dinámica.

Esta idea sitúa la acción humana como parte de un movimiento general del mundo, que se dirige hacia un telos, un fin de máxima plenitud, hacia el que cada ser se esfuerza "a su manera", y nosotros tenemos que hacerlo a la nuestra. Que es la manera de la libertad, y que se plasma además en un esfuerzo de liberación de todo lo demás: trasformar el mundo, para que cada realidad natural pueda dar lo mejor de sí.

Nos hallamos aquí ante un leitmotiv que atraviesa toda la filosofía de Javier Hernández-Pacheco. Un leitmotiv que aparece ya incoado en su primer libro "Subjetividad y reflexión" ${ }^{2}$, publicado en 1983; que alcanza luego un estadio de madurez expositiva en sus brillantes ensayos de la década de los noventa; y que aún seguimos encontrando firmemente sostenido en los libros de su última década.

2 Hernández-Pacheco, Javier, Subjetividad y Reflexión. Tres estudios de antropología fundamental, Publicaciones de la Universidad de Sevilla, Sevilla 1983. Todas las obras de Javier Hernández-Pacheco se encuentran disponibles en: https://personal.us.es/jpacheco/Publicaciones.htm. El leitmotiv que estoy considerando aparece ya con claridad, aunque todavía sin la madurez que luego alcanzaría libros posteriores, en la tercera parte de esta primera obra: "Identidad, historicidad y reflexión". 
Por citar algún texto relevante, podemos quedarnos, por ejemplo, con la formulación que nos ofrece en las páginas finales de su ensayo sobre filosofía de la economía "Elogio de la riqueza"3. (Y ya el lugar que ocupa este pasaje en dicha obra, resulta muy indicativo de la importancia que el tema tiene para él):

[Es preciso] situar el trabajo y la vida económica en el lugar antropológico que le corresponde, a saber, en un contexto más amplio en el que ambos adquieren su sentido, y que viene definido por el fin absoluto de la vida del hombre y de la historia. Es respecto de ese fin, y en tanto que su realización excede las capacidades del hombre, por lo que el trabajo y la vida económica adquieren ahora su sentido propio, a saber, como el modo específicamente humano de contribuir a su realización. Parece que Dios no creó el mundo terminado, sino en proceso evolutivo que tiende progresivamente a su perfección. A este proceso contribuye todo lo que es y existe; y en esta contribución que realizan los pájaros volando, las flores abriéndose en primavera, los niños sonriendo, se despliega, también progresivamente, la plenitud en la que el mundo consiste como obra de Dios. Pues bien, el trabajo, como esfuerzo de mediación entre el espíritu y la naturaleza, entre la libertad subjetiva y la necesidad objetiva, es el modo humano de contribuir.

Y se trata de un modo importante, puesto que al trabajo lo mueve como fin, casi conscientemente, la idea escatológica de una última armonía del universo. Por eso es importante la vida económica. En ella se plenifica el mundo como fruto del esfuerzo; y toda la riqueza creada parece que se pierde sin él. [...] El dinero no da la felicidad, ni el amor de una mujer, ni el cariño de un hijo. Pero repetidamente lo hemos visto: eso que da la felicidad, exige dinero para alcanzar una plenitud que parece sólo incoada en las cosas. Y así el que ama a los suyos sabe que 
esa felicidad se viene abajo si no se construye una casa, si no se les da de comer, si no se les ofrece una educación. Parece, pues, como si el hombre tuviese que hacerse económicamente responsable de un gozo al que hay que buscar albergue. Desde esta perspectiva, es sabio el lenguaje ordinario cuando utiliza la expresión "sacar adelante" [...] para expresar el sentido de la actividad económica. El mundo y el bien que encierra como nuestro gozo está ya hecho, es algo, pues, que hemos de agradecer como un don. Pero ese bien no parece que esté terminado; antes bien, parece que se marchita si no generamos en él las condiciones que exige su prosperidad. $Y$ aquí radica el sentido del trabajo, y con él el de la vida económica.

Quizás es como las herencias. El mundo es un capital que no hemos hecho, que no es fruto de nuestro esfuerzo. Es un regalo, que exige agradecimiento por un lado, y por otro reverencia $[. .$.

Pero también como en las herencias, el agradecimiento por lo recibido y el respeto al pasado, es obligación moral de mantener un legado que se deteriora si no crece. La vida económica adquiere así sentido en el contexto de un mundo que desde su origen consiste en su propia tendencia a la perfección. Ahí se inserta la tarea económica de los hombres, como el afán de hacer prosperar la vida, consciente de que la custodia del mundo implica "sacarlo adelante", hasta que deje de haber hogares sin fuego, niños que no coman y amantes separados por la distancia. 4

La cita es larga pero muy ilustrativa, tanto del papel clave del principio, como de su aplicación a un tema concreto que en este caso es el análisis de la esencia de las actividades económicas. No obstante, la idea general no se circunscribe únicamente a la esfera económica (pese a su indudable importancia), sino que se trata de un esquema de naturaleza ontológica.

La importancia ontológica de ese imperativo de transformación

4 Hernández-Pacheco, Javier, Elogio de la riqueza, Tibidabo Ediciones, Barcelona 1991, p.163-164. 
del mundo para llevarlo a su plenitud fue expuesta por Javier HernándezPacheco en diversos textos, pero quizá en ninguno con tanta claridad y fuerza como en "La conciencia romántica", y sobre todo en el segundo capítulo de ese ensayo, que es la parte que dedica a explicar el origen del idealismo alemán, a partir de la filosofía kantiana, y la conexión del idealismo con el romanticismo.

En el punto de partida de su análisis, aparece la imposibilidad, en el pensamiento kantiano, de lograr una síntesis racional entre Dios, el yo y la naturaleza, que conjugue de modo inteligible estos tres pilares de la ontología, que Hernández-Pacheco identifica con el arkhé [Dios], el logos [yo] y la physis [naturaleza]. En sus propias palabras:

Ahora viene el problema kantiano, que determina el desarrollo posterior del Idealismo. La filosofía trascendental, a mi entender, declara la síntesis final de logos, arkhé y physis como imposible en el ámbito finito de la razón. El entendimiento puede lograr síntesis de conceptos concretos con intuiciones sensibles, siempre de alcance limitado y que constituyen la ciencia natural, tal y como la presenta Newton. Pero la transparencia del mundo para el sujeto, y la comunicación de ambos en lo divino, está fuera del alcance de la razón finita. Y esta síntesis imposible supone la ruptura insuperable de una naturaleza a la que se le escapan sus principios (se ve reducida entonces a "material empírico"); de un sujeto al que se le escapan también las cosas en sí y que, en sí mismo, se convierte en mera condición de posibilidad de la experiencia; de un Dios que está fuera del alcance de la razón subjetiva y cuya presencia en el mundo no es en modo alguno manifiesta. ${ }^{6}$

El problema, es incluso más grave aún, puesto que esta escisión provoca otra más: la del propio sujeto, que por una parte adquiere cierta semejanza con Dios, en tanto que condición de posibilidad de cualquier experiencia, mientras que en otro aspecto no tiene más entidad que la que

5 Hernández-Pacheco, Javier, La conciencia romántica, Tecnos, Madrid, 1995. Todas las obras de Javier Hernández-Pacheco se encuentran disponibles en: https://personal. us.es/jpacheco/Publicaciones.htm .

6 Hernández-Pacheco, Javier, La conciencia romántica, Tecnos, Madrid, 1995, p.40. 
estas experiencias le otorguen, con lo que parece que el propio sujeto, es decir, uno mismo, termina volviéndose incomprensible para sí mismo:

Esto plantea ahora un grave problema en esa teoría de la subjetividad que era el gran logro de la filosofía kantiana. Por un lado, el sujeto es la incondicionada y absoluta condición de toda experiencia. Desde este punto de vista se asemeja a Dios. Pero ese sujeto, tan pronto queremos darle contenido, se disuelve en la pluralidad de sus representaciones y en sí mismo resulta indeterminado. Lo que yo soy en concreto, eso es mi experiencia del mundo; y entonces resulta que ese Yo se encuentra frente a un mundo de objetos en los que tiene su determinación. En sí mismo el sujeto trascendental resulta ser una instancia deducida como condición de la experiencia; y cae entonces en la contradicción de tener en la experiencia las condiciones de su determinación. De este modo, la subjetividad refleja en sí la fractura de naturaleza-Dios-Yo, fracturándose a sí misma en una subjetividad trascendental -en un Yo absoluto-, por un lado, y una subjetividad empírica, por otro, ligada a un acontecer natural del que es en el fondo mero reflejo.?

Llegados a este punto, Javier Hernández-Pacheco realiza una interpretación original, y muy atractiva, de la propuesta de Fichte para salir del atolladero kantiano. Pues según enseñan los manuales de filosofía, Fichte habría propuesto la consideración del "yo" como la auténtica instancia absoluta. Y por tanto aquella de la que depende todo lo demás. Descartando la fractura entre subjetividad trascendental y subjetividad empírica por la vía de considerar los fenómenos como productos del sujeto pensante.

Hernández-Pacheco recoge este planteamiento, pero le da una interpretación en clave moral. Lo cual presenta un doble atractivo: en primer lugar, convierte la absolutización fichteana del "yo", que de entrada parece absurda (...¿cómo aceptar que el "yo" pone el mundo?), en un enunciado que tiene sentido; y en segundo lugar, ese sentido -que es práctico, que es un imperativo ético- nos abre la perspectiva de la misión trasformadora del mundo por medio del trabajo: 
De este modo, la pretensión de absolutez, que en un primer momento el sujeto eleva ante la naturaleza, en la forma de mera protesta, de resistencia a verse arrollado por ella, pasa a ser eficaz reflexión de dicho sujeto en sí mismo por medio de lo que antes era su límite; esa pretensión se convierte en una modificación del mundo por la que éste pasa a ser momento de la autodeterminación del sujeto. La experiencia ya no es mero contacto con lo extraño, y se hace, a medida que la libertad logra su propósito, historia de lo propio. Esta inversión de la naturaleza: de límite a medio, es lo que realiza el trabajo. El trabajo esforzado, lo que Fichte denomina Streben, es el sino de la libertad en medio de ese mundo extraño que es la naturaleza; primero como protesta práctica contra sus limitaciones, y luego, poco a poco, como progresivo proceso de transformación, en el que el hombre va dando a todas las cosas la forma de su libertad. ${ }^{8}$

No es este artículo el lugar apropiado para profundizar en las numerosas vertientes y pormenores que se generan al adoptar esta perspectiva, y que Javier Hernández-Pacheco va analizando en diversos pasajes de sus obras. Si me he detenido a llamar la atención del lector en la raíz metafísica de esta tesis de que estamos llamados a una tarea infinita de transformación del mundo, para hacerlo despliegue de la libertad, es simplemente para mostrar que no se trata de un planteamiento secundario, o de alcance sectorial, sino que brota de las reflexiones más nucleares de su pensamiento. De sus reflexiones sobre Dios, el mundo y el sujeto humano. De su preocupación por la escisión que percibe entre estas instancias, y de su anhelo de unificación en una plenitud final.

Razón por la cual la gran tarea de despliegue de la libertad en el mundo reaparece una y otra vez a lo largo de sus obras, enfocada en cada caso desde una perspectiva acorde al tema que se esté considerando en el libro correspondiente.

Y de ahí que resulte tan conmovedor que el último artículo escrito por Javier Hernández-Pacheco, y publicado ya póstumamente, volviera una vez más sobre este asunto, y lo hiciera en el contexto de la lucha contra la 
enfermedad que poco después acabaría con su vida:

Algunos han caído. Otros caerán. Y todos a nivel planetario, hasta lo profundo de las selvas amazónicas o los asentamientos inuit de Alaska o Yakutia, todavía podemos caer [...] [Pero] hay algo que con toda seguridad ya podemos decir: no sólo que resistiremos, sino que venceremos a la amenaza; que la virología como ciencia va a dar un salto de gigante, lo mismo que la industria farmacéutica; e igual la cooperación internacional, científica, económica y sanitaria. No solamente sabremos más, y estaremos mejor pertrechados ante otras nuevas amenazas que seguro vendrán. También, y ahora gracias a la peste, como otras veces por el hambre y la guerra, y siempre por medio de la muerte, seremos mejores.

Eso va a ocurrir. Con o sin cada uno. Es ley de vida: naturaleza y cultura siempre se superan a sí mismas en su carácter evolutivo y progresista. Por eso, la intención de estas páginas no es proporcionar recetas para superar nuestro actual mal momento. Después de ya no corta vida y de algún estudio, he llegado a la firme convicción de que la filosofía no sirve de preceptora de la existencia, personal o social. [...] Más modestamente le asigna Hegel el papel de lechuza crepuscular, que va siempre por detrás, no dando lecciones, sino aprendiendo del curso de esa historia algo sobre nosotros mismos.

Y eso es lo que queremos hacer aquí: reflexionar, sobre la vida misma, en relación con sus límites, para aprender de nuestros daños, humildad, y esperanza de todos aquellos que ante esos daños no se rinden. ${ }^{9}$

Note el lector que las frases de la cita anterior, las últimas frases que Javier Hernández-Pacheco dejó enviadas para su publicación (y posiblemente las últimas que escribió), poseen el tono de un testamento espiritual. Y es un testamento que apunta precisamente a la tarea descrita en este apartado:

9 Hernández-Pacheco, Javier, "De pandemias y otros daños. Reflexiones optimistas sobre la caducidad de la vida", Raphisa 8, diciembre (2020) p.115-125. El texto citado se encuentra en la página 125 . 
la superación del límite que supone la naturaleza en su negatividad, para trasformar a esta, mediante el esfuerzo humano, en algo mejor. En lo mejor de sí misma. A través de un dinamismo de evolución y progreso, que tiende hacia lo óptimo. Que avanza hacia la plenitud, aunque en este mundo el proceso de avance sea infinito.

La lucha debe darse, y será esforzada. Pero la victoria es previsible, puesto que "es ley de vida: naturaleza y cultura siempre se superan a sí mismas en su carácter evolutivo y progresista".

\section{3- Sed contra}

Al igual que en sus textos, Javier Hernández-Pacheco defendió reiteradas veces en nuestras conversaciones esta visión teleológica de un mundo que se encamina a su plenitud, y de una humanidad que, manos a la obra, contribuye decisivamente en ese dinamismo. Dinamismo de progreso y mejora crecientes, de despliegue de una libertad transformadora (y liberadora) del mundo. Con trabajo, con contratiempos y retrocesos, con daños y con retos, la dirección del proceso de la historia sería positiva. Y el final -infinitamente lejano desde nuestra perspectiva, eso sí- la conversión de toda la naturaleza en un jardín, y toda la vida en un trabajo festivo. Es decir, la recuperación del Paraíso perdido. La recomposición de la intimísima comunidad entre arkhé, logos y physis.

No obstante, yo volvía una y otra vez a plantearle la objeción de que la naturaleza, tal y como viene determinada por las leyes de la física, quizás pueda ser conformada por el hombre hasta cierto punto y durante algún tiempo, por supuesto. Pero que, aunque esto sea así, y aunque la historia humana haya sido hasta ahora una historia de triunfos de la libertad sobre sus límites aparentes, no se trata de un proceso que pueda continuar indefinidamente, y que vaya a ir cada vez a mejor.

Pues, aun suponiendo que el hombre logre mantener bajo control el enorme potencial destructivo de las técnicas que va desarrollando (de la energía atómica, la ingeniería genética, la inteligencia artificial, etc.), lo que quizás sea ya mucho suponer, lo cierto es que la naturaleza tiene inscrita en sus entrañas una tendencia hacia el desorden, que no podrá ser definitivamente vencida, y que, por el contrario, acabará imponiéndose a 
cualquier esfuerzo en contra por nuestra parte ${ }^{10}$.

El problema se encuentra en la termodinámica: La segunda ley de la termodinámica establece que en los sistemas cerrados la entropía (que es una magnitud física asociada con el desorden estructural) siempre tiende a aumentar. De manera que el universo en conjunto - que podemos considerar como un sistema físico, según nos muestra el éxito predictivo de la cosmología actual, y que parece ser el ejemplo más estricto de sistema cerrado - se halla inexorablemente destinado a una progresiva decadencia estructural. Está destinado al desorden más completo.

Esto a primera vista puede resultar chocante y contradictorio con la experiencia. Pues la historia del universo ha dado lugar, entre otras maravillas, a la formación de estrellas, de planetas, y de organismos biológicos. Y los organismos biológicos se encuentran ordenados en grado sumo. Más aún, tanto si consideramos el proceso de desarrollo histórico de estos organismos (la historia de la vida en la Tierra), como si consideramos el proceso de formación de cada organismo particular, lo que se encuentra es un dinamismo de progresiva complejificación y ordenación: de las células primitivas hasta los animales más complejos en un caso, de las células reproductoras a los individuos adultos en el otro.

No obstante, la contradicción es sólo aparente. E indica tan solo que los organismos biológicos no pueden ser sistemas cerrados, sino que deben estar en todo momento intercambiando materia y energía con su entorno. Y haciéndolo de tal modo que el resultado sea el aumento de orden que observamos en ellos, y la conservación luego de ese orden... a cambio, claro está, de aumentar el desorden del entorno. Puesto que la segunda ley de la termodinámica es tan inexorable como el resto de las leyes de la física.

El orden y la estructuración de los organismos es dinámico: sólo se logra mediante la constante interacción de los mismos con su medio, en procesos que tienden a lograr la forma estructural propia de cada organismo,

10 He desarrollado el tema de este apartado por extenso en el primer capítulo de mi ensayo: Soler Gil, Francisco José, Al fin y al cabo. Reflexiones en la muerte de un amigo, Ediciones Encuentro, Madrid 2021. Se trata de un ensayo sobre la muerte y su significado, que he escrito precisamente con ocasión de la muerte de Javier Hernández-Pacheco, y que en parte recoge estos temas controvertidos que discutíamos. A fecha de entrega de este artículo, el libro mencionado se encuentra en prensa. 
y a consolidarla luego frente a la tendencia universal al aumento del desorden. Se trata de una lucha por el orden que además resulta especialmente encarnizada por el hecho de que las piezas estructurales de los seres vivos, las moléculas carbonatadas, tienden a modificarse con cierta facilidad, y por tanto son muy susceptibles a sufrir alteraciones que lo más probable es que vayan en esa dirección general hacia el desorden que marca la segunda ley de la termodinámica.

Por ello, los organismos, y más cuanto más complejos son, cuentan con toda una serie de mecanismos de compensación de los desequilibrios y de reparación e intercambio de sus piezas estropeadas, que les permiten mantener por un tiempo más o menos largo la tensión de esa lucha contra el desorden estructural.

Hasta aquí, la descripción del dinamismo de los seres vivos podría ser contemplada como una confirmación del planteamiento de HernándezPacheco, puesto que tal relato nos informa de la vida como de un proceso de autoafirmación estructural frente al desorden. Y esa autoafirmación mostraría un principio de libertad que luego alcanzará su máxima expresión en el "yo" libre.

Pero lo que ocurre es que esta historia de lucha y autoafirmación tiene un final. Y ese final no es la plenitud, sino la muerte.

Los organismos vivos puede sostener la contienda contra el desorden por un tiempo más o menos largo... pero nunca por un tiempo indefinido. Al final, más tarde o más temprano, la actividad cada organismo biológico colapsa. Este colapso puede ser repentino, debido al efecto de inputs de energía superiores a los que puede acomodar (por ejemplo de energía cinética, recibida en un fuerte impacto, o de energía eléctrica, recibida en una descarga, o de energía térmica etc.), o bien debido a una pérdida de energía súbita, situado en determinados ambientes (por ejemplo excesivamente fríos), etc. Pero aunque esto no ocurra, también se van produciendo desequilibrios paulatinos crecientes debido al aumento de piezas del organismo que dejan de funcionar adecuadamente (desordenadas como consecuencia de una infección, o de una mutación adversa, o del deterioro asociado con errores en las sucesivas replicaciones que tienen lugar en la reproducción celular etc.). Y cuando el número de piezas que no se encuentran bien estructuradas alcanza cierto nivel, algún elemento clave en la estructura global del organismo cesa 
su actividad, y ello acaba finalmente provocando el cese global de la actividad de todo el sistema.

Cuando se llega a este punto, el material que compone el organismo queda, por decirlo así, a la intemperie: expuesto a los procesos de desorden dominantes en la naturaleza. Como consecuencia, las complejísimas moléculas que formaban las células del sistema se van descomponiendo en moléculas más simples (en parte por procesos puramente físicos, y en parte como resultado de la acción de otros organismos que emplean ese material para sus propios dinamismos). El cuerpo se descompone.

Y si esto ocurre a nivel del organismo individual, a nivel cósmico la perspectiva no es diferente:

Extrapolando hacia el futuro la dinámica del universo a gran escala, y haciendo uso además de la física de partículas, y de los datos disponibles sobre la masa total del universo, lo que cabe esperar es que la expansión del mismo continúe indefinidamente. Y que mientras que esto ocurre, dentro de $10^{13}$ años se haya apagado la luz de las últimas estrellas ${ }^{11}$. A continuación, dentro de un margen de aproximadamente 1019 años, la mayoría de los restos de estrellas habrán sido tragados por el agujero negro central de las galaxias, o habrán salido de ellas, y vagarán por el espacio intergaláctico. Durante este proceso, alguna vez habrá algo de luz (cuando choquen dos estrellas enanas marrones), pero a partir de unos 1020 años ya apenas se darán choques. Las estrellas (apagadas) que no hayan salido despedidas de las galaxias irán perdiendo energía por radiación gravitatoria, y terminarán cayendo todas en los agujeros negros centrales. Esto ocurrirá aproximadamente en $10^{25}$ años. Más adelante, en unos $10^{32}$ años (si la teoría de gran unificación es correcta) se habrán descompuesto los protones. Y mucho más adelante aún en el tiempo, hacia 10100 años, se habrán evaporado los agujeros negros, y sólo quedará en el universo una sopa oscura de fotones, neutrinos, electrones y positrones. Pero los positrones y electrones terminarán aniquilándose, y en el universo sólo quedarán fotones sueltos y neutrinos sueltos, cada vez más alejados entre sí, y sin que vuelva a ocurrir nunca nada. Y habremos llegado a lo que se conoce como "muerte térmica del universo".

Por supuesto, tal escenario, sobre todo en sus hitos finales, se basa estrellas enanas perdurará un poco más, hasta agotarse en unos $10^{14}$ años. 
en extrapolaciones muy arriesgadas de la física actual, que podrían resultar incorrectas por muchos motivos distintos - por ejemplo en función de cómo evolucione realmente la constante cosmológica en el futuro lejano-. No obstante, la alternativa que el marco físico actual deja abierta, si ciertos parámetros cosmológicos fueran diferentes a como creemos hoy que son, consiste en el colapso final de toda la materia del universo. Y este escenario resulta al fin y al cabo tan inhóspito como el anterior. De manera que, salvo que nuestra física esté muy equivocada, el futuro a largo plazo del cosmos no es ningún tipo de plenitud, sino la muerte.

¿Y cómo encajar esto con el optimismo teleológico que constituye el marco de la tarea de trasformación del mundo que Javier Hernández-Pacheco ha identificado como imperativo de la subjetividad?

\section{Una propuesta que ya no podremos discutir}

En un informe completo de la controversia entre Javier HernándezPacheco y yo sobre este tema, habría llegado ya el momento de mencionar sus réplicas a la objeción expuesta. Pero esto es algo que no puedo hacer en realidad, puesto que, cuando la muerte cortó el hilo de nuestra charla, seguíamos encontrándonos en ese punto en el que se tiene la impresión de que no nos entendemos, y de que falta algo. Por eso, cualquier intento ahora por mi parte de darle voz concreta a mi amigo y oponente derivaría con facilidad en moneda falsa.

Sólo mencionaré de pasada que una réplica posible tiene que ver con el carácter provisional y falible de la física... Ahora bien, aunque la física sea provisional y falible, resulta difícil encontrar un apoyo más firme que esta ciencia como suelo para una reflexión sobre la naturaleza y su carácter. Con lo que cualquier contraargumento edificado sobre la base de esta provisionalidad tiene que fracasar.

También puede oponerse que el discurso del dinamismo que progresa hacia la plenitud se refiere al mundo de lo humano, y que por tanto es un discurso situado en otro ámbito y a otra escala (de tiempos y dimensiones) que el relato termodinámico. Pero tampoco una respuesta así resultaría satisfactoria, puesto que la tesis de Javier Hernández-Pacheco es ontológica, 
y se refiere a la relación más íntima y de principio entre la libertad del logos y el dinamismo de la physis.

Por tanto, dado que no habíamos llegado todavía a una comprensión conjunta del problema, y que los dos seguíamos dándole vueltas, considero que lo más honrado es evitar poner en boca de Hernández-Pacheco una respuesta con la que quizás él no estuviera de acuerdo.

En lugar de eso, me gustaría ofrecer al lector en este apartado un breve esquema de mis últimas reflexiones sobre la controversia. Reflexiones que tenía pensado discutir con él, en cuanto se presentara la siguiente ocasión propicia. La ocasión ya no va a presentarse en este mundo. Y por eso dejo ahora estos pensamientos en manos del lector, para que los juzgue como estime oportuno.

Podríamos comenzar por Platón -que en filosofía es como empezar por el Génesis-, y en concreto por el genial paralelismo que Platón establece en La República entre el microcosmos del individuo humano y el macrocosmos de la sociedad: entre las dimensiones del alma y los estratos sociales. Al hacer esto, el ateniense inaugura una tradición de establecimiento de puentes analógicos entre el microcosmos de la persona y ámbitos mayores de lo real.

Y en esta misma línea, también por ejemplo San Agustín enlazará en La ciudad de Dios la dinámica de los deseos del corazón humano con la dinámica de las corrientes sociales e históricas: "Dos amores fundaron dos ciudades..."

Cabría por tanto apelar a esta tradición para sugerir que quizás se dé una analogía biográfica entre el hombre y el cosmos como totalidad. Y que así como cada persona nace, se desarrolla, llega a una plenitud en la que debe desplegar todas sus fuerzas y sus iniciativas, y luego declina y muere, igualmente ocurre con el universo en su conjunto.

¿Podría servirnos esta analogía para establecer un acuerdo entre la filosofía de Javier Hernández-Pacheco y la física? Parece difícil, pero quizás no fuera del todo imposible.

Parece difícil, puesto que la cosmovisión de Hernández-Pacheco es la de un mundo que va a más, que va a mejor, que avanza (entre dificultades) hacia una plenitud, si bien nunca terminará de alcanzarla, a este lado del horizonte. Y sólo la gracia, situados ya en el horizonte escatológico, pondrá el infinito restante. 
Es obvio que postular una tendencia sostenida hacia lo mejor no es compatible con el marco físico vigente. Pero en cambio, sí que lo es insistir en la tendencia de la vida hacia esa plenitud, y su lucha por ella. Y, por otra parte, resulta consistente con el planteamiento de Hernández-Pacheco subrayar la necesidad de la gracia, es decir, de una novedad imprevista y que desborda por completo el marco de nuestra finitud, para conseguir el fin que se anhela.

Por eso, juntando todas las piezas, podríamos concluir que es imperativo de nuestra esencia el luchar por la forma, y por una conformación del mundo entero en la línea de la libertad, como proponía HernándezPacheco. Pero que hay que luchar a sabiendas, no ya de que nuestros esfuerzos serán insuficientes, sino de que serán derrotados. De que tenemos por delante una misión que ha de fracasar. Tanto a nivel individual (en nuestra muerte), como a nivel cósmico (en la muerte térmica del universo). De manera que esa derrota nos dejará, al hombre y al cosmos, finalmente inermes, y en espera de una salvación que no puede venir de nosotros mismos.

Cuando en el año 2005 publiqué una colección de textos de diversos autores contemporáneos sobre cosmología y teología, incluí en esa antología un artículo del físico teórico y pastor anglicano John Polkinghorne, que contiene un pasaje muy relevante en este punto. Permítame el lector que lo copie aquí por ello:

De un modo o de otro, el cosmos está condenado a una definitiva inutilidad. Tan cierto es esto como puede serlo el que la vida basada en el carbono resulte haber sido en todas partes un episodio pasajero de su historia. Estos sombríos pronósticos plantean al teísmo cuestiones obvias acerca de cuáles podrían ser las intenciones últimas del Creador para la creación. Ciertamente no alientan ninguna noción de optimismo evolutivo, de una plenitud total a encontrar en el despliegue sin más del presente proceso.

Personalmente, no creo que el conocimiento de la muerte del universo en una escala temporal de decenas de miles de millones de años dé lugar a mayores dificultades teológicas que el conocimiento, incluso más seguro, de nuestras propias muertes en escalas de decenas de años. La cuestión fundamental que se nos plantea es la de si vivimos en un mundo que es un 
cosmos o un caos. ¿Tiene el universo un sentido global, ahora y siempre, o es su historia en definitiva "un cuento contado por un idiota, lleno de sonido y furia, que no significa nada"? Una metafísica naturalista tenderá a coincidir con Steven Weinberg cuando dice que, a la luz de la definitiva inutilidad cósmica, cuanto más comprende el universo, tanto más sin sentido le parece. El naturalismo hace frente a estos hechos con una especie de heroico desafío. Hay una cierta nobleza en este desolado punto de vista, pero yo no creo que nos veamos forzados a adoptarlo.

La muerte, cósmica o humana, es real, pero para el teísta no es la realidad última. La última palabra la tiene Dios, y es la lealtad eterna de Dios la que provoca en las criaturas la esperanza en un destino más allá de la muerte. Si hay alguna esperanza para el universo o para nosotros, ésta sólo puede hallarse en la lealtad eterna de Dios. ${ }^{12}$

Hay que tener en cuenta que Polkinghorne escribe estas reflexiones después de haber resumido en las páginas anteriores toda una serie de indicios favorables a una lectura en clave teísta de la forma de ser del cosmos. Indicios que le impulsan, llegados al punto de la consideración de su final, a la confianza: a esperar un destino más allá de la muerte que podemos anticipar. Y esta es también la perspectiva a la que yo, dentro de mis limitaciones, he ido llegando a lo largo de décadas de estudio sobre el orden natural ${ }^{13}$.

¿Aceptaría Javier Hernández-Pacheco esta modificación en su planteamiento? ¿O la consideraría inasumible desde su idea del papel de la libertad y del sujeto en el orden de lo real?

12 Polkinghorne, John, "Física y metafísica desde una perspectiva trinitaria", en Soler Gil, Francisco José (ed.), Dios y las cosmologías modernas, BAC, Madrid 2005, p.201-222. El texto citado se encuentra en la página 219.

13 El lector interesado puede consultar por ejemplo la segunda parte de Soler Gil, Francisco José, El enigma del orden natural, Editorial Senderos, Sevilla 2020. O el sexto capítulo de mi ensayo: Soler Gil, Francisco José, Al fin y al cabo. Reflexiones en la muerte de un amigo, Ediciones Encuentro, Madrid 2021. 


\section{Epílogo: El valor del fragmento de una discusión}

La controversia con Javier Hernández-Pacheco que he tratado de esbozar en este artículo ha de interrumpirse aquí. Y se interrumpe abruptamente, sin culminación, en fragmento.

Pero quizás esto no sea malo, después de todo. A fin de cuentas, no ha habido en nuestros días mayor defensor del valor del fragmento como lugar manifestación del Absoluto que Javier Hernández-Pacheco. Ciertamente apoyado en los autores del romanticismo temprano, pero con una pasión tal que indica a todas luces su simpatía hacia la tesis de estos:

Lo realmente encantador de la Venus de Milo son los brazos que le faltan; y en la ruina de un castillo perdido en el bosque, entre la hiedra que invade las antiguas estancias, pervive venerable, tan roto como sus muros, como un fantasma, la libertad que lo construyó. Y lo respetuoso es dejar que los fantasmas, fragmentos de sí mismos, habiten sus obras, sin expulsarlos de ellas al sustituir en la reconstrucción su libertad por la nuestra. La ruina nos habla en su lenguaje roto del espíritu antiguo. Está rota porque en parte -en lo que le falta ahora y estaba antes completa - está con los que la construyeron, y en parte está entre nosotros, como vestigio de sí misma y de ese espíritu antiguo. Y si la reconstruyéramos, imitaríamos la forma externa, pero la ruina ya no diría nada, y ese espíritu se perdería para siempre.

Esto no es fantasía poetizante. Mejor dicho, sí lo es; pero en cuanto tal es efectivamente metáfora del Absoluto, su vestigio: fragmento racional de su verdad, del logos que incluye en sí todas las cosas. ${ }^{14}$

También esta discusión es un fragmento de sí misma. Y está rota porque una parte la tengo conmigo, pero otra parte se la ha llevado él. Pero precisamente por eso, ¿acaso no percibe el lector cómo el espíritu de nuestro amigo va recorriendo estas páginas, y va suscitando pensamientos que me sorprenderán de nuevo, y me derrotarán definitivamente? 


\section{Thémata. \\ Revista de Filosofía}

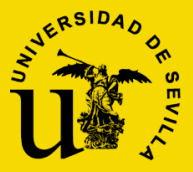

